\title{
Orbital migration in protoplanetary disks
}

\author{
J. C. B. Papaloizou ${ }^{1,2}$ and E. Szuszkiewicz ${ }^{3}$ \\ ${ }^{1}$ Astronomy Unit, Queen Mary, University of London, Mile End Rd, London E1 4NS, England \\ ${ }^{2}$ Department of applied Mathematics and Theoretical Physics \\ Centre for Mathematical Sciences, Wilberforce Road, \\ Cambridge CB3 0WA, United Kingdom \\ ${ }^{3}$ Institute of Physics, University of Szczecin, Wielkopolska 15, 70-451 Szczecin, Poland
}

\begin{abstract}
We review processes leading to the orbital migration of bodies ranging from dust particles up to protoplanets in the earth mass range in protoplanetary disks. The objects most at risk of being lost from the disk have dimensions of the order of metres. To retain these it may be necessary to invoke either strong turbulence or trapping due to special flow features such as vortices. Migration also becomes important for protoplanets with mass exceeding $0.1 M_{\oplus}$. In this case it can also lead to the attainment of commensurabilities for pairs of protoplanets. Such pairs could be left behind after disk dispersal. We review some recent work on the attainment of commensurabilities for protoplanets in the earth mass range.
\end{abstract}

Keywords. planetary systems, planetary systems: formation, planetary systems: protoplanetary disks

\section{Introduction}

The discovery of extrasolar giant planets orbiting close to their host stars (Mayor \& Queloz 1995; Marcy \& Butler 1995, 1998) with periods of a few days has led to an appreciation of the importance of orbital migration during and post formation.

Here we review the processes that lead to orbital migration for objects ranging from meter size up to the several earth mass range. For these masses the migration is entirely determined by the disk in which they are embedded. This is because they are too small to affect the disk density profile sufficiently to provide feedback such as occurs with gap formation in the Jovian mas range. We shall not consider such phenomena here, referring the reader to eg. Lin \& Papaloizou (1993), Kley (2000), Kley, Pietz \& Bryden (2004), Papaloizou (2005) and references therein.

In a non turbulent disk with typically expected parameters, objects exceeding $\sim 1 \mathrm{~km}$. in size, normally considered to be planetesimals, and less than on the order of a lunar mass, orbital migration is unlikely to be important during an expected protostaellar disk lifetime $\sim 10^{7}$ y. For small masses below this lower limit hydrodynamic processes such as gas drag dominate, while for masses exceeding the upper limit gravitational perturbations leading to the excitation of density waves are more important. For objects of about one metre in dimension the migration is potentially so short as to threaten survival but this could be stalled at special locations in the disk. In a turbulent disk this picture is blurred by the effects of potentially strong density fluctuations that can lead to stochastic gravitational forces which in turn may give rise to to a stochastic component to the orbital migration (Nelson \& Papaloizou 2004).

One of the important features of observed exoplanetary systems is the occurrence of mean motion resonances, which are generally supportive of post formation orbital migration. Convergent migration of two objects is believed to be a generic mechanism 
leading to the attainment of commensurabilities in satellite systems within the solar system (see e.g. Goldreich 1965; Gomes 1988). Exoplanetary systems exhibiting low order commensurabilities among giant planets are Gliese 876 (Marcy et al. 2001), HD 82943 (Mayor et al. 2001) and 55 Cancri (McArthur et al. 2001). A natural explanation is that the disk planet interaction produces orbital migration through the action of tidal torques (Goldreich \& Tremaine 1980; Lin \& Papaloizou 1986) which in turn may lead to orbital resonances in a two giant planet system. Several studies have verified this (Snellgrove Papaloizou \& Nelson 2001; Lee \& Peale 2002; Papaloizou 2003; Kley Pietz \& Bryden 2004; Kley et al. 2005).

However, it is likely that planetary systems around other stars may harbor planets with masses in the Earth mass range as well. These should be revealed by future space-based missions, such as Darwin, COROT, Kepler, SIM and TPF.

Accordingly, it is of interest to consider what comensurabilities could be established when two embedded low mass planets migrate in a gaseous disk. We review some recent results of Papaloizou \& Szuszkiewicz (2005) on this problem that indicate that two planets with near equal masses could produce stable low order commensurabilites (e.g. 3:2), more disparate masses could evolve into a stochastic regime in which resonances (e.g. 8:7) are less stable but nonetheless still potentially observable in some cases.

\section{Migration of small bodies}

Small particles are well coupled to the gas and may be modeled as a fluid separate from the gas. For such a two fluid system the equations of motion are (see e.g. Garaud \& Lin 2004).

$$
\frac{\partial \mathbf{v}}{\partial t}+\mathbf{v} \cdot \nabla \mathbf{v}=-\frac{\nabla P}{\rho}+\mathbf{f}-\nabla \Phi-\frac{\rho_{d}\left(\mathbf{v}-\mathbf{v}_{d}\right)}{\rho \tau_{c}}
$$

and

$$
\frac{\partial \mathbf{v}_{d}}{\partial t}+\mathbf{v}_{d} \cdot \nabla \mathbf{v}_{d}=-\nabla \Phi-\frac{\left(\mathbf{v}_{d}-\mathbf{v}\right)}{\tau_{c}}
$$

Here $\mathbf{v}$ and $\mathbf{v}_{d}$ denote the velocities of the gas and dust respectively. The gas density, dust density and a gas pressure are $\rho, \rho_{d}$, and $P$ respectively. The gravitational potential, normaly due to the central mass is $\Phi$. The last term on the right hand side in the equations of motion gives the acceleration due to drag between the gas and solid components. The stopping time $\tau_{c}$ measures the time for a solid particle to relax to the gas velocity. Any additional accelerations acting on the gas, as a result of for example magnetic fields are denoted by $\mathbf{f}$.

For particles that are smaller than a mean free path, that can be estimated to be about $1 \mathrm{~m}$ for conditions appropriate to a minimum mass solar nebula, we have $\tau_{c} \sim$ $\left(4 a \rho_{\alpha}\right) P_{\text {orb }} /(3 \pi \Sigma)$. Here $a$ is the particle radius and $\rho_{\alpha}$ its density. The gas surface density is $\Sigma$ and the orbital period is $2 \pi / \Omega=P_{\text {orb }}$. From this it follows that for bodies of radius $1 m$ the coupling to the gas is good or $\tau_{c}<P_{\text {orb }} /(2 \pi)$, provided $\Sigma \gtrsim 3000 \mathrm{~g} \mathrm{~cm}^{-2}$ at $1 A U$, a condition expected to be satisfied.

For the simple case of a steady axisymmetric disk with $\mathbf{f}=0$ and the gas is in a state of pure rotation with $\mathbf{v}=\left(0, v_{\varphi}(r), 0\right)$ in cylindrical coordinates, one has, neglecting radial drifts, for the rotation of the solid component assumed to be at a rate close to that of the gas

$$
v_{d, \varphi}-v_{\phi}=\frac{-1}{2 \rho \Omega} \frac{d P}{d r}
$$

Drag between the solid and gaseous components then produces aradial drift velocity 
which can be found from the azimuthal component of the equation of motion of the solids in the form

$$
v_{d, r}=\frac{1}{\rho \Omega^{2} \tau_{c}} \frac{d P}{d r}
$$

Note that this depends on the pressure gradient in this case with $\mathbf{f}=0$, but the discussion can be simply generalized to include additional forces.

The normal expectation is that the pressure increases inwards and so when $\mathbf{f}=0$, the expected drift is inwards. Asuming the scale of variation of the pressure to be comparable to the radius, the characteristic magnitude of the drift rate is

$$
v_{d, r} \sim \frac{c^{2}}{r \Omega^{2} \tau_{c}},
$$

where $c$ is the isothermal sound speed.

For bodies marginally coupled to the gas with $\Omega \tau_{c}=1$, being such that $a=(3 \Sigma) /\left(8 \rho_{\alpha}\right)$, the radial drift time is $\sim(1 /(2 \pi))(r \Omega / c)^{2} P_{\text {orb }}$. This is very short approaching $\sim 70$ orbits for aspect ratio $H / r=c /(r \Omega)=0.05$.

\subsection{Gas drag}

The above discussion holds for particles smaller than the mean free path but which can reach sizes up to around one metre. For much larger objects fluid drag operates. We may take this into account by replacing $1 / \tau_{c}$ by $3 C_{D} \rho\left|v_{d, \varphi}-v_{\phi}\right| /\left(4 \rho_{\alpha} a\right)$ in equation (2.2). Here $C_{D}$ is the drag coefficient. For large objects such that the relative gas flow has a Reynold's number greater than of order unity $C_{D}$ is of order unity.

In that case the radial drift rate is modified such that

$$
v_{d, r} \sim \frac{C_{D} \rho c^{4}}{r^{2} \Omega^{3} \rho_{\alpha} a} \sim c \frac{C_{D} \Sigma c^{2}}{2 r^{2} \Omega^{2} \rho_{\alpha} a} .
$$

For $C_{D}=0.2, \rho_{\alpha}=1 \mathrm{~g} \mathrm{~cm}^{-3}, a=10 \mathrm{~m}$ and $\Sigma=10^{3} \mathrm{~g} \mathrm{~cm}^{-2,} v_{d, r}=0.1 c[c /(r \Omega)]^{2}$. The radial flow time scale is then $(5 / \pi)\left(r \Omega / c_{s}\right)^{3} P_{\text {orb }}$. For $c /(r \Omega)=0.05$, this is $\sim 15000$ orbits.

The above discussion indicates potentially serious survival problems for objects about one metre in size. A possibility is that the lifetime in this size range is short, a situation that might arise if the sedimenting solids undergo gravitational instability (e.g. Goldreich \& Ward 1973). However, it is now believed that a sedimenting disk, even though initially laminar, is subject to sufficient turbulence (e.g. Weidenschilling 1980, 2003) to prevent enough sedimentation to allow gravitational instability to occur.

An alternative possibility is that special regions of the flow are significant. In particular particles may accumulate at pressure extrema where the relative drift between gas and dust is expected to be small. In fact one generally expects attraction to pressure maxima for particles tightly coupled to the gas. If gas elements remain near such a maximum, then because the equations of motion for solid particles and gas coincide at an extremum in pressure, even particles not very tightly coupled to the gas can remain there also. Note that for the case when pressure varies only with radius, because the radial drift velocity is proportional to the pressure gradient, a pressure maximum is a stable attractor, while a minimum is unstable. This discussion emphasizes the potential significance of special locations in the disk for accumulating solids. The importance of the role of vortices in this respect has also been considered (e.g. Barge \& Sommeria 1995).

For larger objects the drift time is $\propto 1 / a$, and this becomes comparable or longer than expected gas disk lifetimes for objects larger than a few kilometres independently of flow features provided they are sufficiently smooth. However, as objects approach 


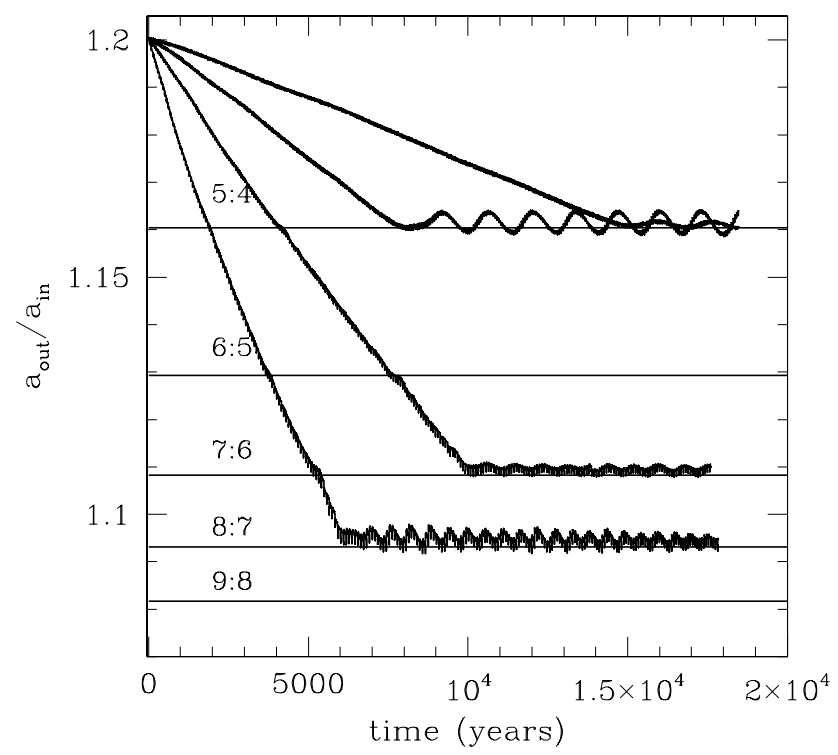

Figure 1. The evolution of the ratio of semi-major axes of two planets with masses $m_{1}=4 M_{\oplus}$ and $m_{2}=1 M_{\oplus}$. Starting from the lower curve and going upwards the curves correspond to the initial surface density scalings $\Sigma_{0}=4 \Sigma_{1}, \Sigma_{0}=2 \Sigma_{1}, \Sigma_{0}=\Sigma_{1}$, and $\Sigma_{0}=0.5 \Sigma_{1}$ respectively.

$\sim 0.1 M_{\oplus}$, direct gravitational perturbation of the disk becomes more important than local hydrodynamic processes such as drag in determining radial drift. This we consider below.

\subsection{Migration of low mass planets}

For low mass planets the disk undergoes small linear perturbations that induce density waves that propagate away from the planet. The angular momentum these waves transport away results in a potentially rapid orbital migration called type I migration (Ward 1997). In this type of migration, when the disk is laminar and inviscid, the planet is fully embedded and the surface density profile of the disk remains approximately unchanged. This, as well as the well known density wakes associated with the disk planet interaction, is illustrated in calculations such as those illustrated in Figures 1 and 2 below. The timescale of inward migration on a circular orbit can be derived from a linear response calculation and estimated for a disk with constant surface density to be given by (see Tanaka Takeuchi \& Ward 2002)

$$
\tau_{r}=\left|\frac{r}{\dot{r}}\right|=W_{m} \frac{M_{*}}{m_{p}} \frac{M_{*}}{\Sigma r^{2}}\left(\frac{c}{r \Omega}\right)^{2} \Omega^{-1}
$$

Here $M_{*}$ is the mass of the central star, $m_{p}$ is the mass of the planet orbiting at distance $r$ and the numerical coefficient $W_{m}=0.3788$.

It is important to note that type I migration appropriate to a laminar disk may lead to short migration times in standard model disks, that may threaten the survival of protoplanetray cores (Ward 1997). For $m_{p}=M_{\oplus}$, and $\Sigma=200 \mathrm{gm} \mathrm{cm}^{-3}$, the inward migration time at $5.2 \mathrm{AU}$ is $\sim 10^{6} \mathrm{y}$, being inversely proportional to mass. Thus bodies smaller than $1 \mathrm{~km}$. and larger than about $\sim 0.1 M_{\oplus}$ could be lost through migration within potential gas disk lifetimes of up to $\sim 10^{7}$ y (see e.g. Papaloizou \& Larwood 2000).

However, in a disk with turbulence driven by the magnetorotational instability, the migration may be stochastic and accordingly less effective (Nelson \& Papaloizou 2004). 

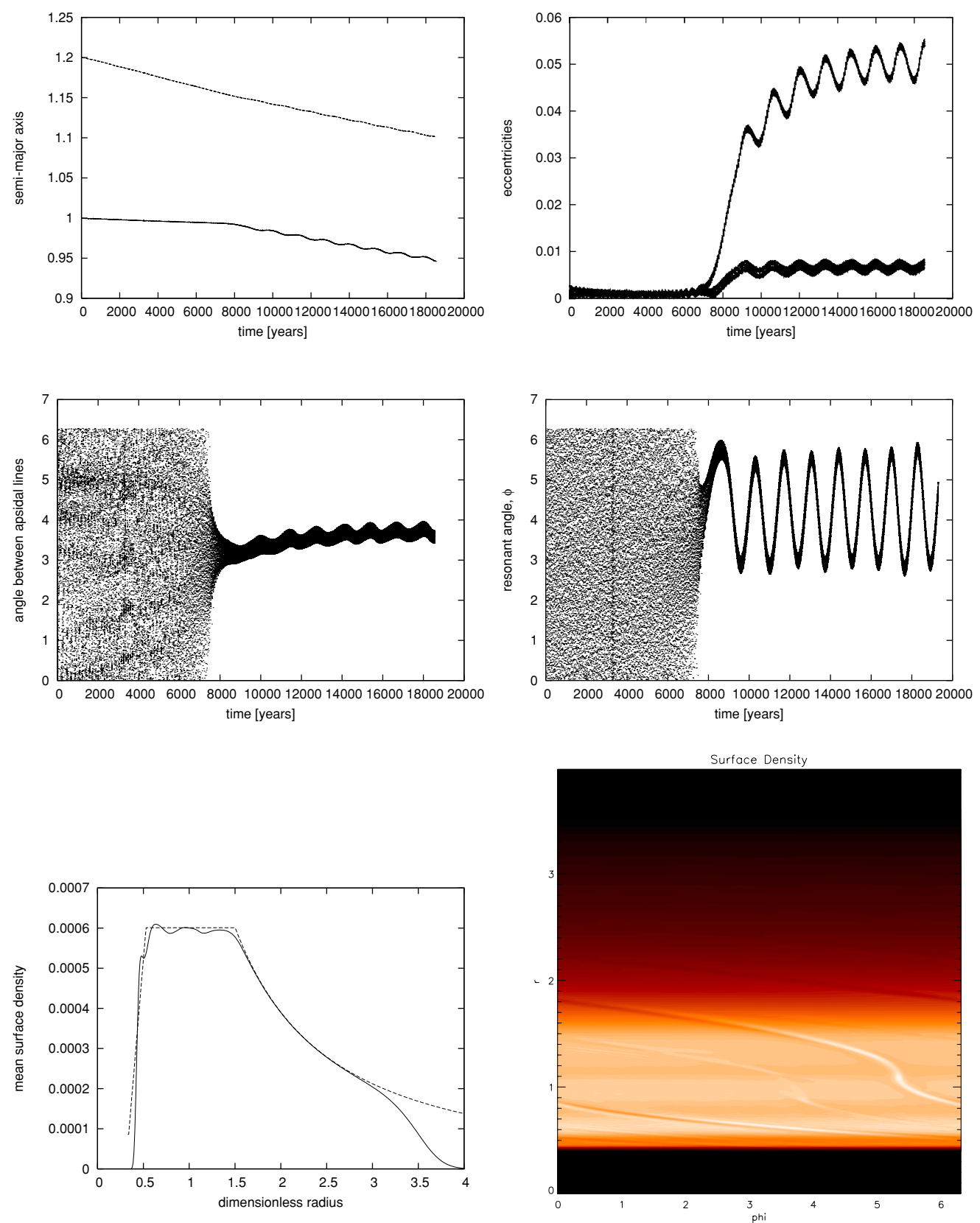

Figure 2. The evolution of the semi-major axes, eccentricities, angle between apsidal lines and the resonant angle for planets with masses, $m_{1}=4 M_{\oplus}$ and $m_{2}=M_{\oplus}$ migrating towards a central star, embedded in a disk with initial surface density scaling $\Sigma_{0}=\Sigma_{1}$ (four upper panels). In this and similar plots, the resonant angle is appropriate to the commensurability attained at the end of the simulation. The mean surface density profile of the disk near the end of the simulation (solid line) and the initial surface density profile (dashed line) and a surface density contour plot are given in two lower panels. 
This effect could be important for stalling migration during some evolutionary phases. Nonetheless there is considerable uncertainty as to the extent of turbulent regions in the disk resulting from uncertainties in the degree of ionization (e.g. Fromang, Terquem \& Balbus, 2002) so that type I migration appropriate to a laminar disk may operate in some regions. We also note that because it is inversely proportional to the disk surface density, the migration time becomes long in low surface density regions. Hence type I migration in a laminar disk may be significant in some regions of protoplanetary disks. Hence the recent study by Papaloizou \& Szuszkiewicz (2005) of the commensurabilites that could be realized by a pair of interacting planets migrating in a laminar disk with masses in the $M \oplus$ range. The density waves excited by a low mass planet with small eccentricity also lead to orbital circularization (Artymowicz 1993; Papaloizou \& Larwood 2000) at a rate that can be estimated to be given by (Tanaka \& Ward 2004)

$$
t_{c}=\frac{\tau_{r}}{W_{c}}\left(\frac{c}{r \Omega}\right)^{2} .
$$

Here, the numerical coefficient $W_{c}=0.289$.

\subsection{Eccentricity damping due to gas drag}

We consider a body of mass $m_{\alpha}$, density $\rho_{\alpha}$ and radius $a$ moving in an eccentric orbit relative to the disk gas with speed $v$. The equation of motion for $v$ is

$$
m_{\alpha} \frac{\mathrm{d} v}{\mathrm{~d} t}=-\pi a^{2} C_{D} \rho v^{2},
$$

This leads to a relative velocity damping time, $t_{g d}=|v /(d v / d t)|$, equivalent to

$$
\frac{1}{\Omega t_{g d}}=\frac{(36 \pi)^{1 / 3} \Sigma C_{D}}{8 \rho_{\alpha}^{2 / 3} m_{\alpha}^{1 / 3}} \frac{e r}{H},
$$

where we have set $v=e r \Omega$ with $e$ corresponding to the orbital eccentricity. This gives in cgs units

$$
t_{g d}=5.27 \times 10^{3}\left(\frac{m_{\alpha}}{10^{18}}\right)^{1 / 3} \frac{H}{e r}\left(\frac{50 \rho_{\alpha}^{2 / 3}}{\Sigma C_{D}}\right)\left(\frac{r}{1 \mathrm{AU}}\right)^{3 / 2} \mathrm{yr} .
$$

Note that this time can be small for small masses, which is related to the need to evolve fast through the size range where decoupling from the gas first occurs.

\subsection{Relative importance of gas drag and disk tides}

Because of the dependence on protoplanet mass, gas drag is more effective for smaller masses while disk tides take over for larger masses. The transition mass, which is also the mass for which the damping time is a maximum, can be estimated by equating the gas drag time given by equation (2.11) with the eccentricity decay time resulting from disk tides given by equation (2.8). This gives

$$
\frac{m_{\alpha}}{\mathrm{M}_{\oplus}}=5.2 \times 10^{-4} \frac{f_{s}^{15 / 8} C_{D}^{3 / 4}}{\rho_{\alpha}^{1 / 2}}\left(\frac{H / r}{0.05}\right)^{3}\left(\frac{H / r}{e}\right)^{-3 / 4}\left(\frac{r}{5.2 \mathrm{AU}}\right)^{-3 / 2} .
$$

Although there is significant sensitivity to disk location and aspect ratio, the crossover mass is characteristically on the order of a few percent of a lunar mass. Thus gas drag will be the dominant eccentricity damping process when planetesimals first form. 
However, if many larger mass cores form and then interact during the later stages of core accumulation, disk tides will dominate.

\section{Convergent migration of planets in the earth mass range and their attainment of commensurabilities}

It is expected from equation (2.7) that two planets with different masses will migrate at different rates. This has the consequence that their period ratio will evolve with time and may accordingly attain and, in the situation where the migration is such that the orbits converge, subsequently become locked in a mean motion resonance (Nelson \& Papaloizou 2002; Kley et al. 2004).

In the simplest case of nearly circular and coplanar orbits the strongest resonances are the first-order resonances which occur at locations where the ratio of the two orbital periods can be expressed as the ratio of two consecutive integers, $(p+1) / p$, with $p$ being an integer. As $p$ increases, the two orbits approach each other and the strength of the resonance increases. In addition, the distance between successive resonances decreases as $p$ increases. The combination of these effects ultimately causes successive resonances to overlap and so, in the absence of gas, leads to the onset of chaotic motion.

Resonance overlap occurs when the difference of the semi-major axes of the two planets is below a limit with half-width given, in the case of two equal mass planets, by Gladman (1993) as

$$
\frac{\Delta a}{a} \sim \frac{2}{3 p} \approx 2\left(\frac{m_{\text {planet }}}{M_{*}}\right)^{2 / 7},
$$

with $a$ and $m_{\text {planet }}$ being the mass and semi-major axis of either one of them respectively. Thus for a system consisting a two equal four earth mass planets orbiting a central solar mass we expect resonance overlap for $p \gtrsim 8$. Conversely we might expect isolated resonances in which systems of planets can be locked and migrate together if $p \lesssim 8$.

However, note that the above discussion does not incorporate the torques producing convergent migration or eccentricity damping and thus may not give a complete account of the forms of chaos that might be expected. Kary, Lissauer \& Greenwig (1993) have considered the case of small particles migrating towards a much more massive planet and indeed conclude that chaotic behaviour is more extensive in the non conservative case.

In a stochastic regime, long term stable residence in commensurabilities is not possible. However, a system can remain in one for a long time before moving into another higher order commensurability. Furthermore detailed outcomes are very sensitive to input parameters. Slight changes can alter the sequence of commensurabilities a system resides in making the issue of their attainment acquire a probabilistic character. Although there may be islands of apparent stability, a system in this regime may ultimately undergo a scattering and exchange of the orbits of the two planets so that we should focus on stable lower degree commensurabilities as being physically possible planetary configurations. The arguments given above suggest that these must have $p \lesssim 8$. Our calculations indicate that the limit is even smaller.

Hydrodynamic simulations of disk planet interactions, in which the disks are modelled as flat two dimensional objects with laminar flow governed by the Navier Stokes equations and which incorporate a migrating two giant planet system which evolves into a 2:1 commensurability have been performed by Kley (2000) and Snellgrove et al. (2001) and successfully applied to the GJ876 system. In addition to performing additional simulations, Papaloizou (2003) has developed an analytic model describing two planets migrating in resonance with arbitrary eccentricity. In this model the eccentricities are 
determined as a result of the balance between migration and orbital circularization. However, disk tides were considered to act on the outer planet only. Capture of giant planets into resonance has also been recently investigated numerically by Kley et al. (2004).

\section{Numerical simulations of migrating planets in resonance}

Papaloizou \& Szuszkiewicz (2005) performed simulations of two interacting planets embedded in and interacting with an accretion disk. These simulations are similar in concept to those of Snellgrove et al. (2001) and references therein, where the resonant coupling in the GJ876 system induced by orbital migration was studied.

One can a system of units in which the unit of mass is the central mass $M_{*}$, the unit of distance is the initial semi-major axis of the inner planet, $r_{2}$, and the unit of time is $2 \pi\left(G M_{*} / r_{2}^{3}\right)^{-1 / 2}$, being the initial orbital period of the inner planet.

In these dimensionless units the inner boundary of the computational domain was at $r=r_{\text {min }}=0.33$, and the outer boundary at $r=r_{\max }=4$. The equation of state is locally isothermal with aspect ratio $H / r=0.05$ and the explicit viscosity is set to zero. The radial boundaries were open and $n_{r}=384$, and $n_{\varphi}=512$ equally spaced grid points were adopted in the radial and azimuthal directions respectively.

Two planets with masses $m_{1}$ and $m_{2}$ orbiting a central star with mass $M_{*}$ were considered. The two embedded planets began on circular orbits at radii $r_{1}$ and $r_{2}$ respectively. The gravitational potential due to the planets was softened with softening parameter $b=0.8 H$. This gives type I migration rates in reasonable agreement with Tanaka,Takeuchi \& Ward (2002) (Nelson \& Papaloizou 2004). The initial disk surface density was taken to be given by

$$
\Sigma(r)=\left\{\begin{array}{c}
0.1 \Sigma_{0}\left(15\left(r-r_{\min }\right) / r_{\text {min }}+1\right) \text { if } r \geqslant r_{\text {min }} \text { and } \\
r \leqslant 8 r_{\min } / 5 \\
\Sigma_{0} \text { if } r>8 r_{\min } / 5 \text { and } r<4.5 r_{\min } \\
\Sigma_{0}\left(4.5 r_{\min } / r\right)^{1.5} \text { if } r \geqslant 4.5 r_{\min }
\end{array}\right.
$$

where $r_{\min }$ is the inner edge of the computational domain. The planets begin in the flat part of this profile.

The use of dimensionless units enables the results to be scaled so that they apply to different radii and corresponding initial surface densities.

However, in order to make things definite $r_{2}$ was taken to be $5.2 \mathrm{AU}$, but nonetheless the time measured in years.

We adopt a fiducial value of the surface density scaling $\Sigma_{0}=\Sigma_{1}=2 \cdot 10^{3}\left(5.2 \mathrm{AU} / \mathrm{r}_{2}\right)^{2}$ $\mathrm{kg} / \mathrm{m}^{2}$. Thus when $r_{2}=5.2 A U$, this corresponds to a typical value attributed to the minimum mass solar nebula at 5.2AU.

\subsection{Attainment of Commensurability}

The interaction of the planets with the disk leads to spiral wave excitation, energy and angular momentum exchange between with the disk, orbital migration and eccentricity damping. When the migration of the two planets is convergent, by which we mean that the ratio of their semi-major axes increases, first order commenurabilities where the period ratio is $(p+1) / p$ for some integer $p$ will inevitably be approached. As $p$ increases, the planets become closer together. As a result of this it is possible that the planets become locked in mean motion resonances (e.g. Goldreich 1965, Lee \& Peale 2002; Ferraz-Mello, Beaugé \& Mitchchenko 2003; Beaugé Ferraz-Mello \& Mitchchenko 2003) such as 5:4, 


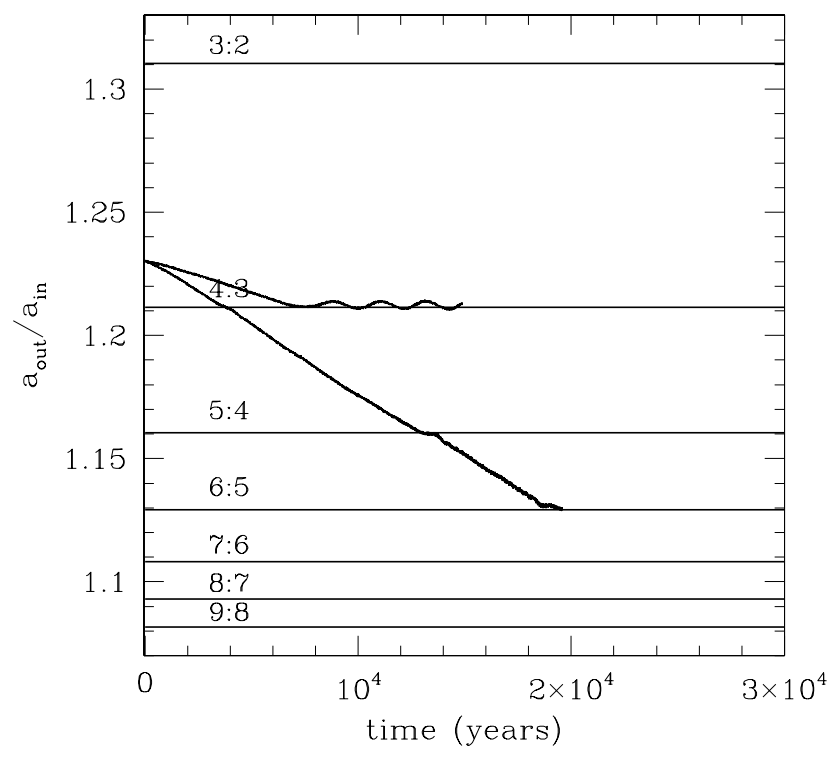

Figure 3. The semi-major axis ratio for $m_{1}=4 M_{\oplus}$ and $m_{2}=1 M_{\oplus}$ with surface density scaling parameter $\Sigma_{0}=0.5 \Sigma_{1}$ - upper curve and $\Sigma_{0}=\Sigma_{1}$ - lower curve).

$6: 5,7: 6,8: 7, \ldots,(p+1): p, \ldots$ and subsequently migrate together maintaining the commensurability for a considerable period of time or perhaps indefinitely, depending on how close the system is to an unstable stochastic regime.

Papaloizou (2003) has given a simple approximate analytic solution for two migrating planets locked in a 2:1 $(p=1)$ commensurability and Papaloizou \& Szuszkiewicz (2005) have generalized this so it is applicable to the commensurabilities with larger $p$ discussed here. Mutual resonant interaction between the planets tends to cause the eccentricities of both planets to grow with time, an effect which is counterbalanced by the effects of interaction with the disk leading to an equilibrium condition.

Close to a balanced $p+1: p$ resonance, the resonant angles $\phi=(p+1) \lambda_{1}-p \lambda_{2}-\varpi_{1}$, $\psi=(p+1) \lambda_{1}-p \lambda_{2}-\varpi_{2}$ and $\varpi_{1}-\varpi_{2}$, with $\lambda_{i}$, and $\varpi_{i}$ denoting the mean longitude and longitude of periapse of planet $i$, librate about equilibrium values which can be near to 0 or $\pi \bmod 2 \pi$. Simulations for planets in the earth mass range indicated libration about a value closer to $\pi$ than 0 .

Which resonance (or value of $p$ ) is established depends on the rate of convergent migration. The resonances become stronger as $p$ increases, so that increasing the relative migration rate tends to cause the attainment of larger values. However, if $p$ becomes too large ( $p \gtrsim 8$ for the planet masses considered) the motion of the planets becomes chaotic (see equation (3.1) making commensurabilities unstable in the long term (although a system may remain in the vicinity of one for a considerable time) while introducing sensitivity of the orbital evolution to the initial conditions. In such situations a scattering may ultimately occur that interchanges the positions of the planets. Thus very high surface densities that are associated with very rapid migration rates are not conducive to the attainment of stable commensurabilities.

We performed simulations to investigate the dependence of attained commensurabilities on on the rate of convergent migration and initial conditions. To do this a pair of planets with masses $m_{1}=4 M_{\oplus}$ and $m_{2}=1 M_{\oplus}$ were set up in circular orbits with $r_{1} / r_{2}=1.2$ in disks with $\Sigma_{0}=0.5 \Sigma_{1}, \Sigma_{0}=\Sigma_{1}, \Sigma_{0}=2 \Sigma_{1}$ and $\Sigma_{0}=4 \Sigma_{1}$. 

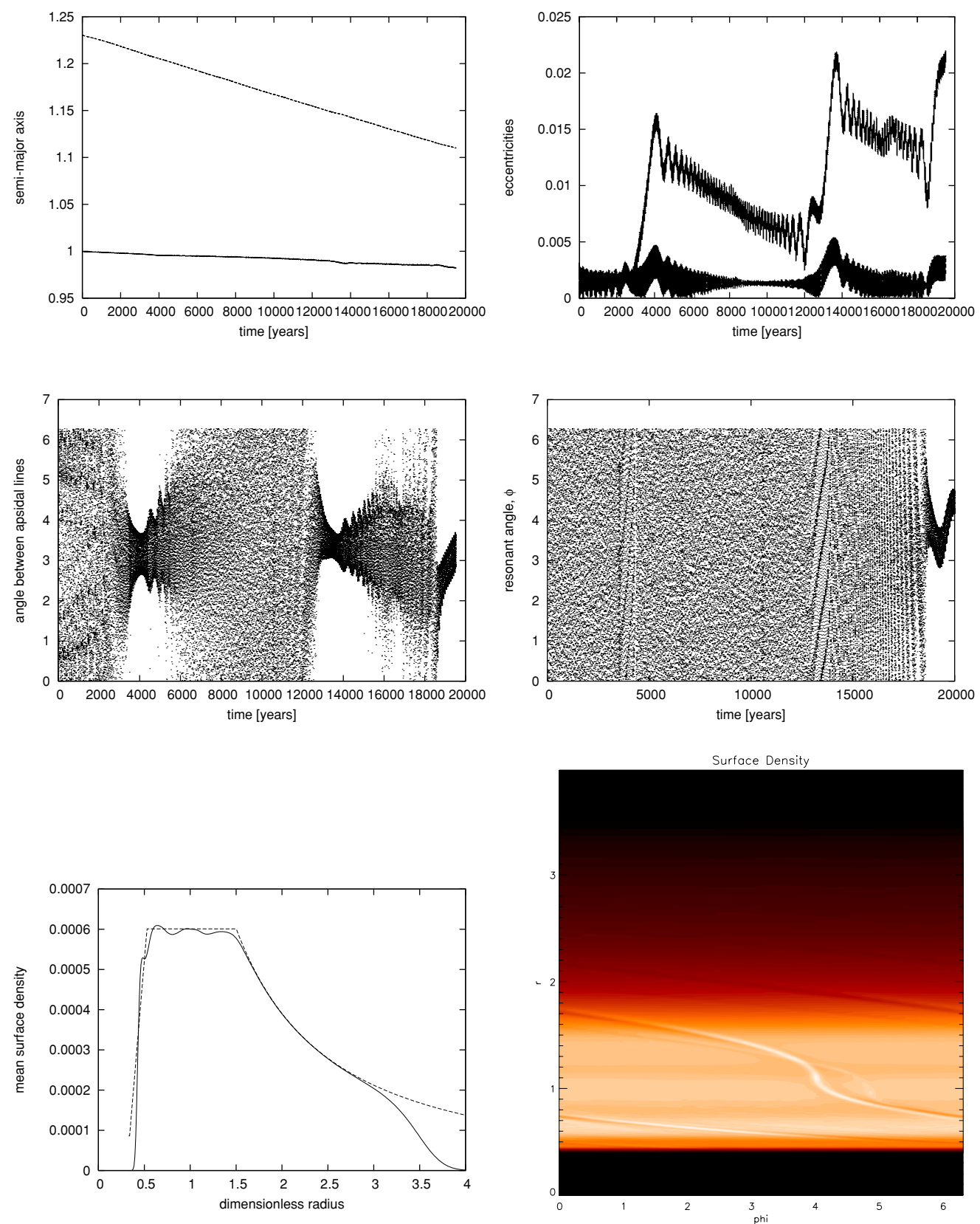

Figure 4. As for Figure 2 but when the planets are initiated with $a_{1} / a_{2}=1.23$.

The subsequent evolution shown in Figures 1 and 2 indicates that a resonance is established such that $p$ increases with the value of $\Sigma_{0}$ or equivalently the rate of migration as expected. The planets in the disk with $\Sigma_{0}=4 \Sigma_{1}$ become trapped in 8:7 resonance. For $\Sigma_{0}=2 \Sigma_{1}$ a $7: 6$ resonance is attained. For $\Sigma_{0}=\Sigma_{1}$ and $\Sigma_{0}=0.5 \Sigma_{1}$ a $5: 4$ commensurability results.

The evolution for the simulation that started with $\Sigma_{0}=\Sigma_{1}$ leads to attainment of a 5:4 commensurability at around 8000 years. Subsequently the inner planet semi-major 


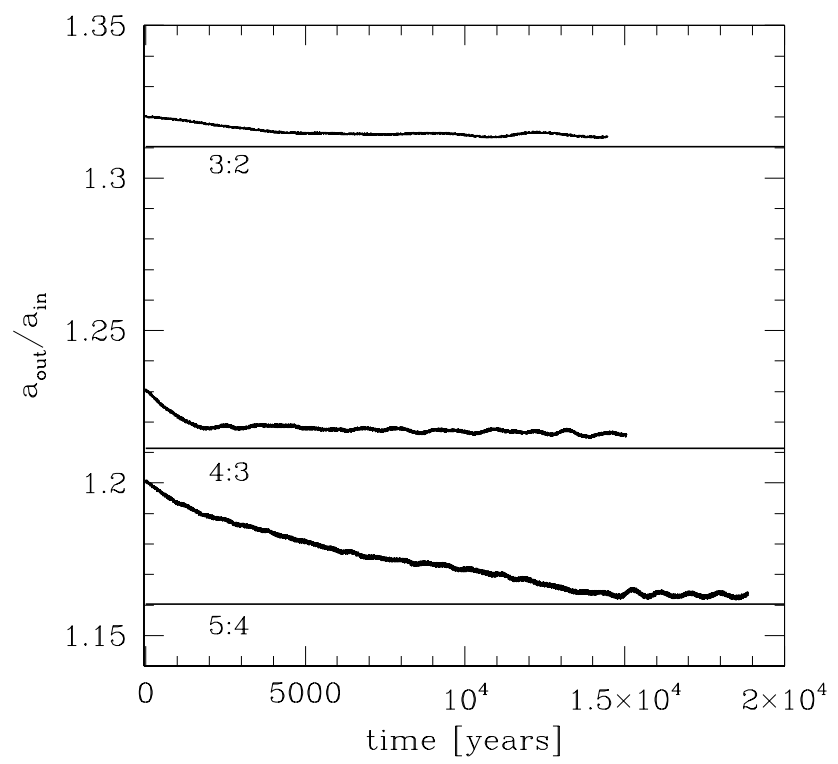

Figure 5. The semi-major axis ratio when $m_{1}=m_{2}=4 M_{\oplus}$ with $\Sigma_{0}=\Sigma_{1}$ (uppermost curve) and with $\Sigma_{0}=4 \Sigma_{1}$ (two lower curves).

axis changes in a characteristic oscillatory manner. These oscillations are clearly visible in Figure 2.

The eccentricity ratio at the end of the simulation is $e_{1} / e_{2}=0.18$. The eccentricities are still growing. The angle between the apsidal lines starts to librate at time 8000 years around $180^{\circ}$ and at time 18000 years oscillates around $212^{\circ}$. The resonant angle excursions have large amplitude and seem to grow with time, which might indicate that the planets will not remain in this resonance.

\subsection{Dependence on the initial separation of the planets}

The dependence of the behaviour at a commensurability on initial conditions and the general lack of stability at some resonances is indicated by the fact that when the ratio of the initial semi-major axes is changed from 1.2 to 1.23 , the case with $\Sigma_{0}=\Sigma_{1}$ passes through the 4:3 and 5:4 resonances and becomes trapped in the 6:5 resonance (see Figure 3).

The passage of the planets through the 4:3 resonance and the temporary trapping in the 5:4 resonance can be seen in Figure 4. At time 4000 years there is a rapid increase in the eccentricities of both planets followed by a slower decrease. The angle between the apsidal lines also shows the expected behaviour during resonance crossing. When the system starts with a smaller initial planet separation as illustrated in Figure 2 the effect of a prior resonance passage before approaching and becoming relatively stably trapped in the 5:4 resonance is not present. So it is likely that the mutual interaction of planets during resonance crossing influences their subsequent evolution and that these planets are either close to or in the chaotic regime.

\subsection{Equal mass planets and lower $p$ commensurabilities}

As described above, in equilibrium the resonant interaction must balance the tendency towards convergent migration of the two planets. As indicated by use of equation (2.7), 

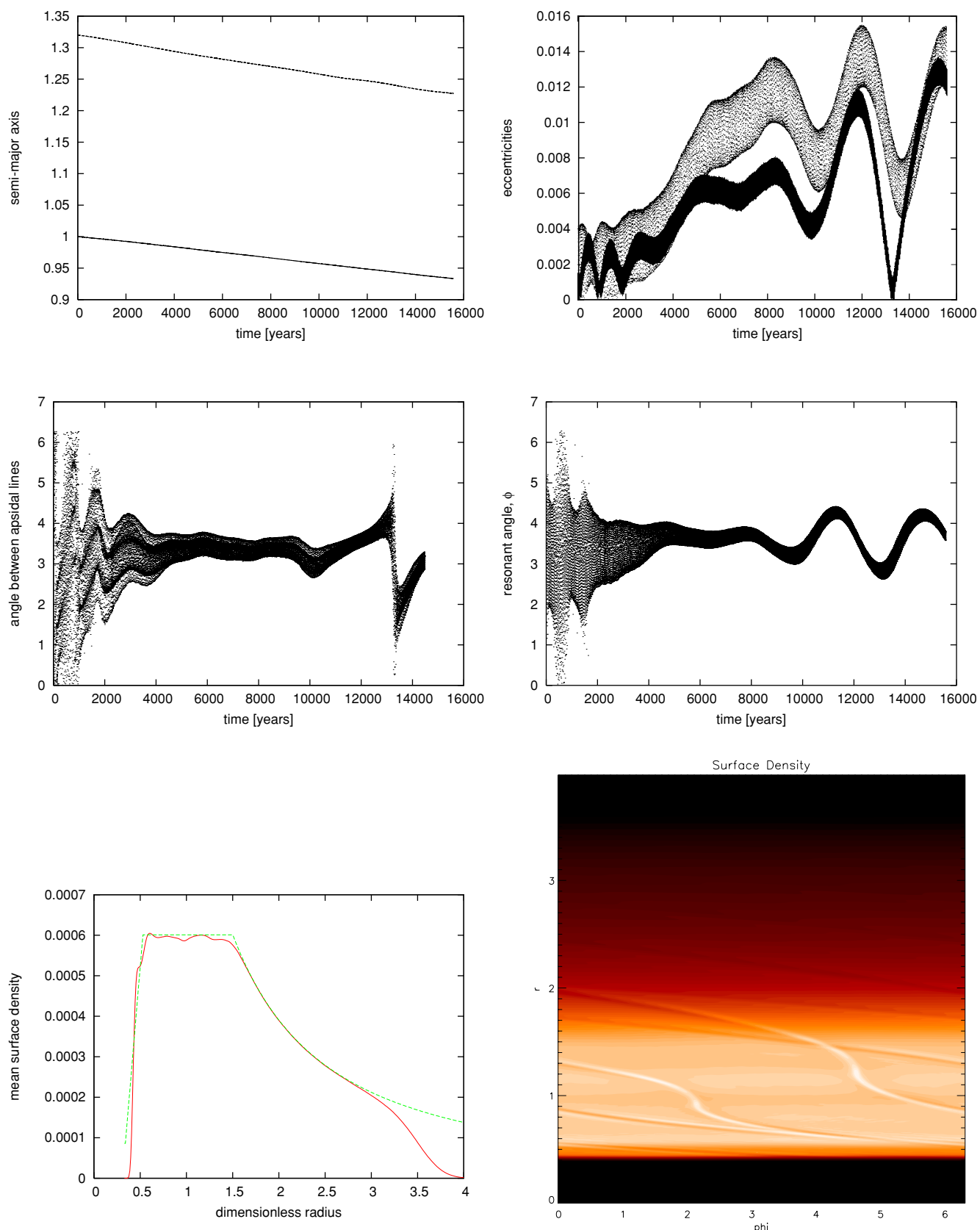

Figure 6. The evolution of semi-major axes, eccentricities, angle between apsidal lines and resonant angle for two planets with equal mass, $m_{1}=m_{2}=4 M_{\oplus}$, migrating towards a central star and embedded in a disk with $\Sigma_{0}=\Sigma_{1}$. (four upper panels). The mean surface density profile of the disk near the end of the simulations (solid line), together with the initial surface density profile (dashed line) and a surface density contour plot near the end of the simulation are given in the lowest left and right panels respectively. 
this is smaller when the planets have the same mass. In fact the ratio of the migration rates for equal masses at a commensurability is found to be $((p+1) / p)^{1 / 3}$. Thus the rate of convergence is about ten percent of the absolute migration rate even for $p=3$. Accordingly, lower degree commensurabilities and less tendency to be driven into a chaotic state might be expected in this case when compared to the situation where the inner planet has significantly smaller mass.

In a simulation study, two planets of mass $4 M_{\oplus}$ were initiated close to $3: 2$ resonance with $r_{1} / r_{2}=1.32$ in a disk with $\Sigma_{0}=\Sigma_{1}$. The evolution of the semi-major axis ratio for the planet orbits is shown in Figure 5. We also performed simulations for the same masses starting with $r_{1} / r_{2}=1.23$ in a disk with $\Sigma_{0}=4 \Sigma_{1}$ and $r_{1} / r_{2}=1.2$ in a disk with $\Sigma_{0}=4 \Sigma_{1}$.

In each of these cases, the planets become trapped in the nearest available resonance, itself a good indication of stability. The evolution of the equal mass pair of planets is shown in Figure 6. The planets attained 3:2 resonance after about 4000 years, had increasing eccentricities until after about 8000 years $e_{1} \sim 0.013$ and $e_{2} \sim .007$. Subsequently the eccentricities oscillated with the resonant angle oscillating around $200^{\circ}$.

\subsection{N-body integrations}

In order to study migrating planets with a wider range of masses and disk surface densities, Papaloizou \& Szuszkiewicz (2005) studied the evolution of migrating pairs of planets using an N-body code. Migration and circularization at rates governed by equations (2.72.8) are incorporated by including appropriate non conservative terms in the equations of motion. For details of the procedure see Snellgrove et al. (2001), Nelson \& Papaloizou (2002), or Kley et al. (2004). The outcome of these simulations could be well matched to those of the hydrodynamic simulations over their relatively short run times, for appropriate choices of $W_{m}$ and $W_{c}$ which were quite close to those suggested by Tanaka,Takeuchi \& Ward (2002) and Tanaka \& Ward (2004). However, there was a strong sensitivity of outcomes such as the duration for which a commensurability with specified $p$ was maintained to these. This is symptomatic of chaotic motion as has been described by for example (Kary, Greenwig \& Lissauer, 1993). However, in view of the fact that some of the sensitivity to initial conditions could be a manifestation of a sequence of passages close to hyperbolic fixed points, the extent of significant chaotic motion is unclear.

\section{Discussion}

We have reviewed processes that can lead to orbital migration in a protostellar disk. Masses ranging from those just decoupling from the gas, typically of radius $1 \mathrm{~m}$. for standard protoplanetary disk parameters migrate quickly unless they are at special disk locations such as pressure extrema.

Objects in ranging from $\sim 1 \mathrm{~km}$. to about a lunar mass are relatively safe from migration in a laminar disk over expected protoplanetary disk lifetimes.

Recent work of Papaloizou \& Szuszkiewicz (2005) has shown that masses in the earth mass range can undergo convergent type I migration. When masses are disparate this normally leads to resonances such as 8:7 or 7:6 for which the motion might be stochastic. However for more equal masses comensurabilities such as 3:2 can be obtained which are much more stable. 
In a well known example of such a system, the two largest mass planets orbiting a millisecond radio pulsar PSR B1257+12, are close to a 3:2 commensurability (Konacki \& Wolszczan 2003). Such a resonance could be formed and maintained through the convergent migration discussed here, with the planets later moving slightly out of resonance. Studies of commensurabilities have already been applied successfully to analyse the motion of both commensurable pairs of giant planets and the pulsar planets, they proved to be a powerful tool in that context. They have the potential to be similarly useful in our search for Earth-like planets in other systems. Detection of such resonances should yield useful information about orbital migration occuring during and post planet formation.

\section{References}

Artymowicz, P. 1993, ApJ, 419, 155

Barge, P. \& Sommeria, J. 1995, A\&A, 295

Beaugé, C., Ferraz-Mello, S., \& Mitchchenko, T.A. 2003, ApJ, 593, 1124

Ferraz-Mello, S., Beaugé, C., \& Mitchchenko, T.A. 2003, Cel. Mech. and Dynam. Astron. 87,99

Fromang, S., Terquem, C., \& Balbus, S.A. 2002, MNRAS, 329, 18

Gladman, B. 1993, Icarus, 106, 247

Garaud, P. \& Lin, D.N.C. 2004, ApJ, 608, 1050

Goldreich, P. 1965, MNRAS, 130, 159

Goldreich, P. \& Tremaine, S. 1980, ApJ, 241, 425

Goldreich, P. \& Ward, W.R. 1973, ApJ, 183, 1051

Gomes, R.S. 1998, AJ, 116, 997

Kary, D.M., Lissauer, J.J., \& Greenzweig, Y. 1993, Icarus, 106, 288

Kley, W. 2000, MNRAS, 313, L47

Kley, W., Peitz, J., \& Bryden, G. 2004, A\&A, 414, 735

Kley, W., Lee, M.H., Murray, N., \& Peale, S.J. 2005, A\&SA, 437, 727

Konacki, M. \& Wolszczan, A. 2003, ApJ, 591, L147

Lee, M.H. \& Peale, S.J. 2002, ApJ, 567, 596

Lin, D.N.C. \& Papaloizou, J.C.B. 1986, ApJ, 309, 846

Lin, D.N.C. \& Papaloizou, J.C.B. 1993, in: E.H. Levy \& J.I. Lunine (eds.), Protostars and Planets III (Tucson: University of Arizona Press), p. 749

Marcy, G.W. \& Butler, R.P. 1995, 187th AAS Meeting baas, vol. 27, p. 1379

Marcy, G.W. \& Butler, R.P. 1998, Annu. Rev. Astron. Astr., 36, 57

Marcy, G.W. Butler, R.P., Fischer, D., Vogt, S.S., Lissauer, J.J., \& Rivera, E.J. 2001, ApJ, 556, 296

Mayor, M. \& Queloz, D. 1995, Nature, 378, 355

Mayor, M., Udry, S., Naef, D., Pepe, F., Queloz, D., Santos, N.C., \& Burnet, M. 2001, A\&\&A, 415,319

McArthur, B.E., Endl, M., Cochran, W.D., Benedict, G.F., Fischer, D.A., Marcy, G.W., Butler, R.P., Naef, D., Mayor, M., \& Queloz, D. 2001, ApJ, 614, L296

Nelson, R.P., Papaloizou, J.C.B., Masset, F., \& Kley, W. 2000, MNRAS, 318, 18

Nelson, R.P. \& Papaloizou, J.C.B. 2002, MNRAS, 333, 26

Nelson, R.P. \& Papaloizou, J.C.B. 2004, MNRAS, 350, 849

Papaloizou, J.C.B. 2003, Cel. Mech. and Dynam. Astron., 87, 53

Papaloizou, J.C.B. 2005, Cel. Mech. and Dynam. Astron., 97, 33

Papaloizou, J.C.B. \& Larwood, J.D. 2000, MNRAS, 315, 823

Papaloizou, J.C.B. \& Szuszkiewicz, E. 2005, MNRAS, In press

Snellgrove, M., Papaloizou, J.C.B. \& Nelson, R.P. 2001, A\& A, 374, 1092 
Tanaka, H., Takeuchi, T., \& Ward, W.R. 2002, ApJ, 565, 1257

Tanaka, H. \& Ward, W.R. 2004, ApJ, 602, 388

Ward, W.R. 1997, Icarus, 126, 261

Weidenschilling, S.J. 1980, Icarus, 44, 172

Weidenschilling, S.J. 2003, Icarus, 165, 438 